Journal of

Applied Crystallography

ISSN 0021-8898

Received 4 April 2003

Accepted 23 October 2003

C 2004 International Union of Crystallography Printed in Great Britain - all rights reserved

\section{Characterization of dislocations in protein crystals by means of synchrotron double-crystal topography}

\author{
B. Capelle, ${ }^{a *}$ Y. Epelboin, ${ }^{\text {a J. Härtwig, }}{ }^{\text {b }}$ A. B. Moraleda, ${ }^{\mathrm{c}}$ F. Otálora ${ }^{\mathrm{c}}$ and V. \\ Stojanoff ${ }^{d}$
}

\begin{abstract}
aLMCP, UMR 7590 CNRS, Université P. M. Curie, case 115, 75252, Paris, France, ${ }^{\mathbf{b}}$ ESRF, BP220, 38043 Grenoble Cedex, France, 'LEC (IACT), Campus Fuentenueva (Fac. Ciencias), 18002 Granada, Spain, and ${ }^{\mathbf{d}}$ Brookhaven National Laboratory, National Synchrotron Light Source, Upton, NY 11973, USA. Correspondence e-mail: capelle@Imcp.jussieu.fr
\end{abstract}

\begin{abstract}
Hen egg-white lysozyme (HEWL) crystals have been studied by means of double-crystal synchrotron topography. The crystals reveal a number of features that are quite well known in hydrothermally grown inorganic crystals: dislocations, growth bands and growth sector boundaries. Dislocations in the $\langle 110\rangle$ sectors have been characterized as edge dislocations with Burgers vector parallel to the c axis. They are distinguishable only under weak beam conditions. The presence of edge dislocations shown in this paper is consistent with the spiral growth steps previously reported. This spiral growth on protein crystals has been observed many times by surface techniques.
\end{abstract}

\section{Introduction}

The increasing quality and size of tetragonal HEWL solutiongrown crystals has naturally led to the study of their perfection by various methods. Among them, X-ray diffraction topography has revealed different kinds of defects: line defects, and two-dimensional (growth sectors, boundaries, etc.) as well as three-dimensional defects. Topographic studies have shown that their quality is rather good. The characterization of crystal perfection is necessary to evaluate their mosaic spread because diffraction conditions depend on the perfection, enabling crystals to diffract to high resolution. Characterization is also of interest in the study of the growth conditions of macromolecular crystals, to obtain a better understanding of the effects of the growth process on the quality of the crystals produced, and to envisage new methods and growth conditions to produce better crystals.

$\mathrm{X}$-ray topographic studies, which have been applied to proteins, can be split into two basic techniques (Bowen \& Tanner, 1998): white-beam topography (Izumi et al., 1996, 1999) and double-crystal topography (Fourme et al., 1995; Stojanoff \& Siddons, 1996; Stojanoff et al., 1997; Dobrianov et al., 1999; Otálora, Garcìa-Ruiz et al., 1999; Otálora, Cappelle et al., 1999; Otálora, Cavira et al., 1999). The observation of the internal contrast of individual Laue spots (white-beam topography) presents a number of advantages. Easy to record, it allows in a single experiment a number of images to be recorded corresponding to various reciprocal reflection vectors. It is well adapted to qualitative analysis but a fine analysis of the contrast is not easy because many wavelengths are present at the same time in the incident beam. Another problem is the possibility of greater damage because of the higher irradiation. Double-crystal topography, i.e. limited wavelength bandwidth and limited divergence, provides a much better resolution and enables an easier quantitative analysis of the images.

The present work is the first part of a long-term project at the ESRF, one of the aims of which was to determine how a more sensitive method, namely double-crystal topography using a third-generation synchrotron, would improve the resolution and the contrast in order to characterize the defects better (Baruchel et al., 2002).

\section{Experimental methods}

Hen egg-white lysozyme (Sigma L-6876) was chosen as a model protein owing to its high resistance to radiation damage, the feasibility of growing large rather perfect crystals, and easy handling. Crystal growth conditions at low supersaturation were selected to produce large crystals of the tetragonal polymorph $\left(P 4_{3} 2_{1} 2, a=7.91 \mathrm{~nm}, c=3.79 \mathrm{~nm}\right)$. Batch growth experiments were set by mixing stock solutions in all reactors (Eppendorf tubes) in adequate proportions to obtain a starting solution containing $50 \mathrm{mg} \mathrm{ml}^{-1}$ lysozyme, without further purification, $2.5 \% w / v \mathrm{NaCl}, 0.1 \% \mathrm{D} 5$ agarose, and $50 \mathrm{mM}, \mathrm{pH}=4.5, \mathrm{Na}$ acetate buffer. These conditions simultaneously ensure the low nucleation density required to obtain a few large crystals, and small growth rate and rate of change of supersaturation. Since the aim of this study was to study the nature of growth defects, no special provision was made to control the temperature very carefully.

The crystals obtained display large $\{110\}$ faces forming a prism limited at each end by four $\{101\}$ pyramidal faces (Fig. 1). The crystal forms, crystallographic axes and typical faces are shown on the right-hand side of Fig. 1. The crystal volume is composed of 12 growth sectors produced by the advance of eight pyramidal faces equivalent to $\{101\}$ by symmetry and 

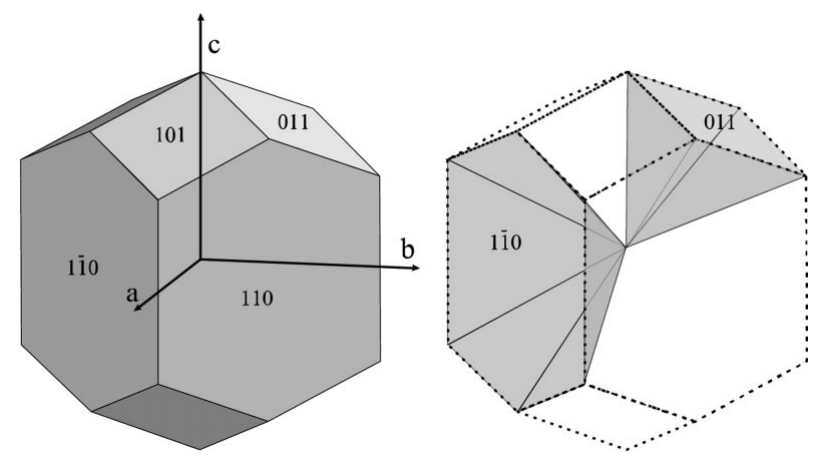

Figure 1

Sketch of a typical tetragonal lysozyme crystal.

four prismatic faces equivalent to $\{110\}$ by symmetry. One such growth sector of each of the two types is shown on the lefthand side of Fig. 1. During their growth, each of these faces generates a well defined growth sector.

The four-circle diffractometer on beamline ID19 of the European Radiation Synchrotron Facility (ESRF) (Baruchel, 2000) was employed to record rocking curves and X-ray topographic images from the samples described above. The beam size at the sample position, $150 \mathrm{~m}$ from the undulator source, was reduced to $1 \mathrm{~mm}^{2}$ by several slit assemblies located in the beamline. The samples were mounted in a ringshaped holder on a standard goniometer head and were preoriented with the help of a telescope. For each sample, several standard oscillation images were recorded on a MAR Research 345

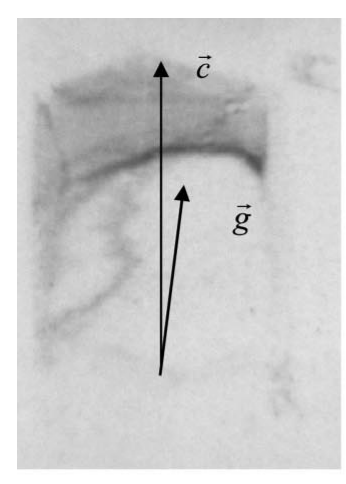

(a)

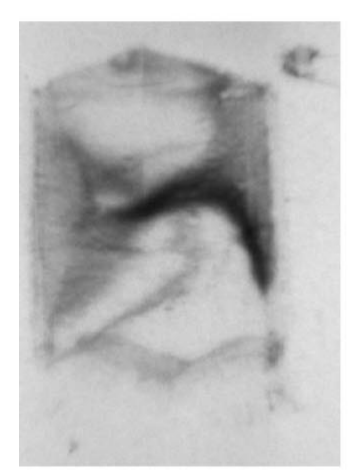

(d)

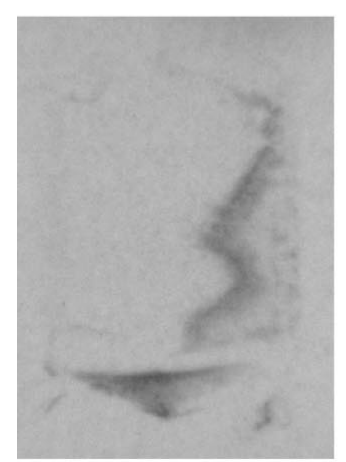

(g)



(b)

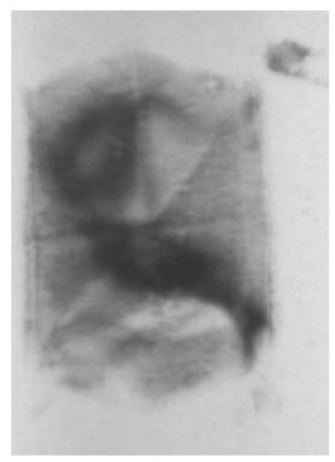

(e)

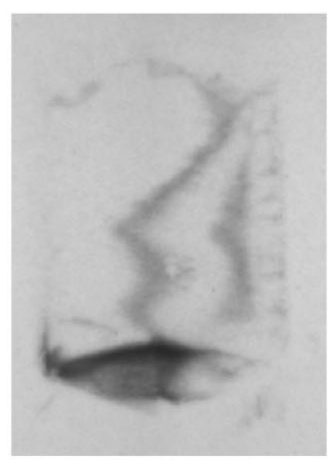

(h)

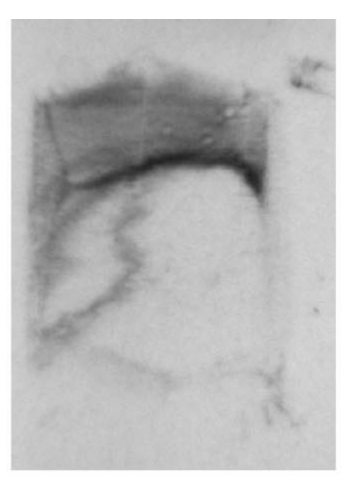

(c)

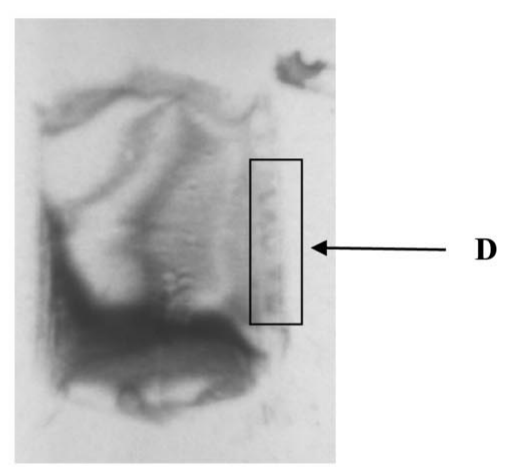

(f)

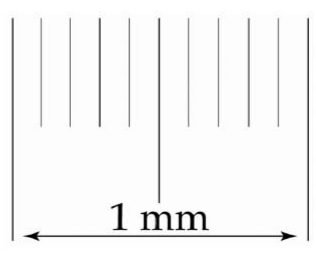

Figure 2

Series of topographs with variation of the Bragg angle of $4 / 1000^{\circ}$. Note the edge dislocations in $(f)$ indicated by arrow D. image-plate detector and indexed with the help of the $H K L$ suite of programs (Otwinowski \& Minor, 1997; Carter \& Sweet, 1997). This procedure was used to choose the appropriate reflection; this is especially useful in order properly to characterize defects such as dislocations using extinction conditions. The rocking-curve measurements of the selected reflection were carried out in a double-crystal configuration with an $\mathrm{Si}$ (111) double-crystal monochromator and by rocking the samples through $\omega$. Topographic images were recorded along the rocking curves on Kodak Industrex SR5 film. The experiment and exposure times varied from a few seconds up to $100 \mathrm{~s}$. The images were later digitized. Ten crystals were studied during the run.

\section{Defect analysis}

A series of topographs is shown in Fig. 2, recorded for different Bragg angles with an angular step of $4 / 1000^{\circ}$. The film to crystal distance was $16.5 \mathrm{~cm}$ and the horizontal width of the image is about $1 \mathrm{~mm}$. The $\mathbf{c}$ direction lies vertically along the prismatic faces. The reflection vector $\mathbf{g}$ is approximately vertical, $10^{\circ}$ to the right of the $\mathbf{c}$ axis.

The first main feature is that not all of the crystal is in reflection at the same time. This arises from the longrange curvature of the crystal: the top part is in reflection in Fig. 2(a), while the bottom part is in reflection in Fig. 2(h), which corresponds to a radius of curvature of about $5 \mathrm{~m}$. 
Fig. 2(e) presents the usual contrast along the limits between growth sectors, as usually seen in solution-grown crystals. The contrast arises from small changes in the parameters and disorientation between the sectors.

In the series of images Figs. $2(d)$ to $(h)$, one may notice the appearance of a contrast similar to a series of horizontal dislocations (marked D in Fig. 2f). They are most visible in the later images when most of the crystal is out of reflection. In image Fig. $2(d)$, for instance, the individual contrasts merge and give rise to a continuous black zone. These lines start from a growth surface parallel to the $\{110\}$ face and resemble the usual contrast of dislocations generated after the restart of the growth.

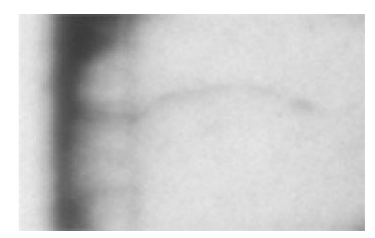

\section{Figure 3}

Top part of image: refraction of a dislocation through a restart surface. Bottom part of image: creation of a bundle of dislocations starting from a defect along this surface.

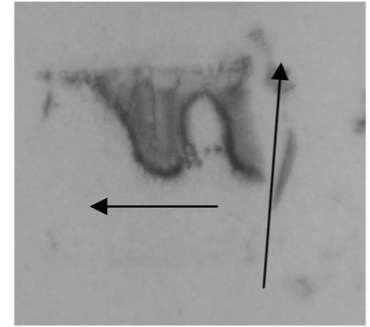

(a)

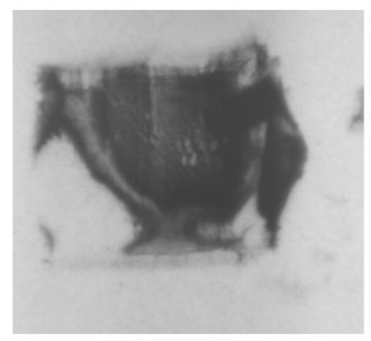

$(d)$

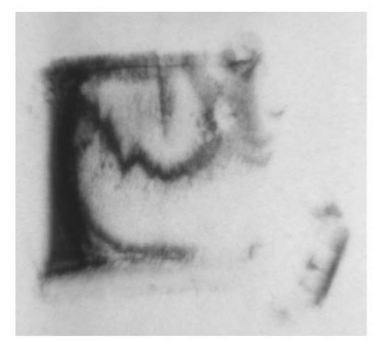

(g)

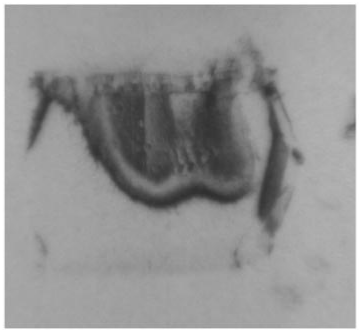

(b)

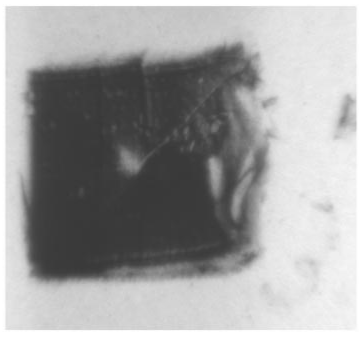

(e)

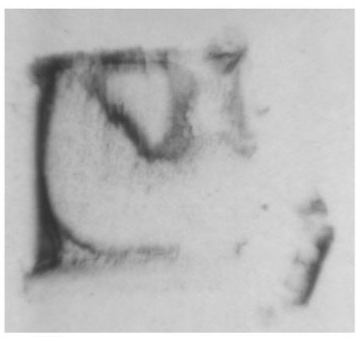

(h)

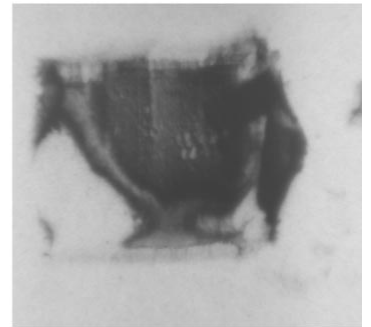

(c)

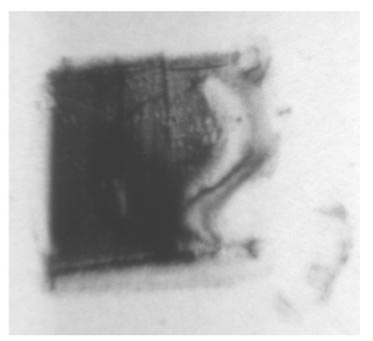

$(f)$

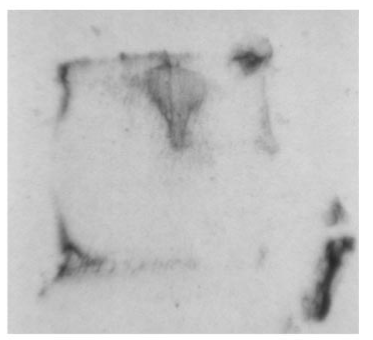

(i)
Figure 4

Series of topographs with $5 / 100^{\circ}$ change in Bragg angle. Same crystal as in Fig. 2 rotated by $90^{\circ}$. The c axis lies horizontally.
Fig. 3 is another example: a curved dislocation crosses a regrowth boundary and changes direction. In the bottom part, a point defect along this surface gives rise to a bundle of dislocations. These features are very common in solutiongrown crystals (Gits-Leon \& Lefaucheux, 1978) but have never been observed in proteins. Such dislocations appear during the growth.

Fig. 4 presents a series of topographs from the same crystal rotated by $90^{\circ}$. The $\mathbf{c}$ axis lies horizontally and the reflection vector $\mathbf{g}$ remains approximately vertical. The images show the same long-range contrast and the prismatic growth sectors remain visible mainly in images Fig. 4(b) to 4(e), where most the crystal is in reflection. The regrowth boundary parallel $\{110\}$ face remains visible but the bundle of dislocations ng from this striation is out of contrast. The remaining stresses in the crystal.

Fig. 5 presents an image of the same crystal in another orientation: the crystal is now oriented with the $\mathbf{c}$ axis towards the beam. The pyramidal sectors at the end of the crystal are fully visible. The dislocations observed in Figs. 2 and 3 are invisible. They never appear in the full set recorded for various departures from the exact Bragg angle.

Based on these three series of topographs, we may now assert that these lines are edge dislocations. The lines lie approximately along the $\langle 110\rangle$ direction, not far from the normal to the faces. Any other direction would be energetically unfavourable. Their Burgers vector lies along the [001] direction.

Defect images, such as dislocations, may be invisible in selected reflections. The conditions for no contrast, i.e. the extinction conditions for dislocations, correspond to reflection planes that are not deformed or are slightly deformed by the defect. For dislocations they can be expressed as $(\mathbf{g}, \mathbf{b}, \mathbf{l})=0$ and $\mathbf{g} \cdot \mathbf{b}=0$, where $\mathbf{I}$ lies along the dislocation line and $\mathbf{b}$ is the Burgers vector of the dislocation. A minimum of contrast arises whenever $\mathbf{g} \cdot \mathbf{b}=0$ and this condition is usually sufficient to characterize edge or mixed dislocations. In the present case the dislocations are visible in Fig. 2 and vanish in Figs. 4 and 5 , which suggests that the Burgers vector lies along the $\mathbf{c}$ direction. The energy of a dislocation is proportional to the square of the value of the Burgers vector, $b^{2}$. Since the parameters of HEWL are $e_{1}=e_{2}=7.91 \mathrm{~nm}$, $e_{3}=3.79 \mathrm{~nm}$, a Burgers vector along $\mathbf{c}$ seems very reasonable because it corresponds to the lowest energy. If the Burgers vector was either $\mathbf{a}$ or $\mathbf{b}$, the 
energy of the dislocation would be four times greater. Since the dislocations lie near the $\langle 110\rangle$ directions, they are mixed dislocations, being mostly edge dislocations.

The ratio of the energies of a screw and an edge dislocation is $E_{\text {screw }} / E_{\text {edge }}=1-v$, where $v$ is the Poisson coefficient of the material. In a soft material, such as HEWL, $v$ is never high; thus the difference in energy between an edge and a screw dislocation with the same Burgers vector is small. The Burgers vector of a dislocation is a combination of the parameters of the crystal: $\mathbf{b}=\sum_{i=1,3} m_{i} \mathbf{e}_{i}$, where each $m_{i}$ is an integer. In the present case, the lines lie approximately along the $\langle 110\rangle$ direction. If the dislocation was a screw dislocation, its Burgers vector would be parallel to $\langle 110\rangle$ when the Burgers vector of an edge dislocation is parallel to $\langle 001\rangle$, and would thus be much shorter. Since the energy is proportional to $b^{2}$, the energy of an edge dislocation is much lower than the energy of a screw dislocation and the appearance of screw dislocations

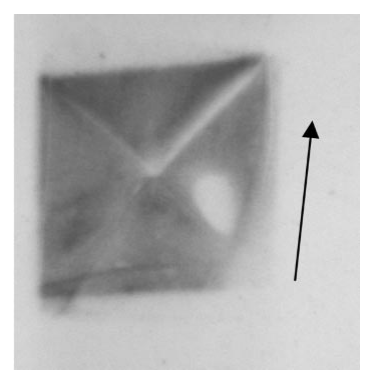

Figure 5

Same crystal as in Fig. 2 but rotated so that the $\mathbf{c}$ axis is perpendicular to the plane of the figure.

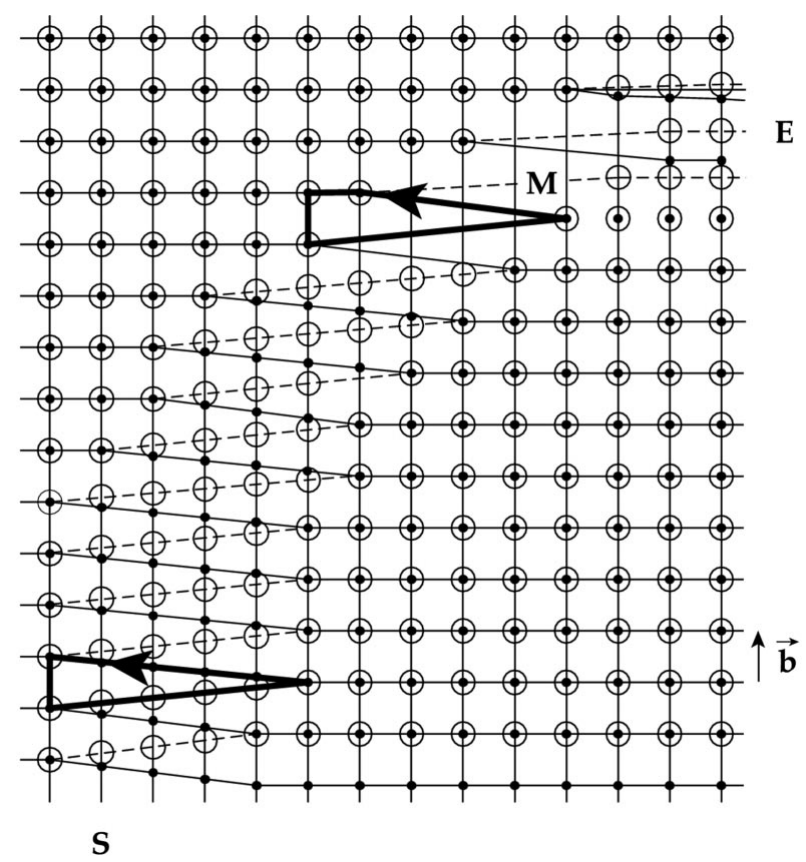

Figure 6

Schematic drawing of a curved dislocation. The dots represent nodes over the plane of the drawing, while the circles represent nodes below. The Burgers vector $\mathbf{b}$ is vertical. At point $\mathrm{S}$ the dislocation is a screw dislocation, at point $\mathrm{E}$ it is an edge dislocation, while at point $\mathrm{M}$ a mixed dislocation is shown. during the growth is unfavourable from the energetic point of view. The dislocations visible in Fig. 2 grew together with the crystal since they start from a growth surface and the direction of the lines lies very near the normal to the surface to minimize their length and thus their total energy. Taking into consideration the supersaturation at which the crystal has grown, the presence of screw dislocations is not needed to ensure continuous growth and, in any case, the repeatable spiral steps needed for growth at low supersaturation can also be present at non-normal outcrops of edge dislocations. This fact can influence the reported change in lysozyme crystal habit (i.e. relative growth rate of different faces) as a function of supersaturation (Durbin \& Feher, 1986). A similar behaviour is already known for mineral crystals grown in solution, such as potash alum (Gits-Leon et al., 1978; Lefaucheux et al., 1973) where the number of edge dislocations participating in the growth is much larger than the number of screw dislocations.

Izumi et al. (1999) have observed screw dislocations in HEWL using diffraction conditions corresponding to Fig. 5. For the same geometry, we do not observe any dislocations; thus such dislocations do not exist in our crystals. On the other hand, if edge dislocations like those present in Fig. 2 exist in the crystals of Izumi et al., they would be invisible in their experiment because of the condition of minimal contrast $\mathbf{g} \cdot \mathbf{b}$ $=0$. Looking at the images in Fig. 2, one may notice that the contrast of individual dislocations is visible only when most of the crystal is out of reflection, far from the maximum of the Bragg peak (images Fig. $2 c$ and Figs. $2 f$ to $2 h$ ). When most of the crystal is in reflection, a continuous dark contrast only is noticeable (image Fig. 2e). The width of an image arises mainly from its direct image (Authier, 1987) and is proportional to the derivatives of $\mathbf{g} \cdot \mathbf{u}$, where $\mathbf{u}$ is the displacement induced by the dislocation. The width of the image is proportional to $\mathbf{b}$, which means, in the present study, that the images are very large. It is maximum under the exact Bragg condition and decreases when the crystal is set far away. Thus, in images Figs. 2(d) and 2(e), individual contrasts overlap and the dislocations cannot be seen individually. Far from Bragg condition, under weak-beam conditions, the width of each contrast becomes narrow enough so that each dislocation can be distinguished. With soft materials with large unit cells, such as HEWL, recording a full set of double-crystal topographs around the Bragg peak is the best method to be sure to characterize individual dislocations.

Different authors have tried to characterize the defects by surface studies (Malkin et al., 1999; Yoshizaki et al., 2001). The presence of growth spirals at the crystal surface has often been ascribed to the existence of screw dislocations, although Strunk (1996) has warned against such an attribution.

Many arguments about the existence of screw dislocations in macromolecular crystals are based on the observation of growth spirals by surface techniques such as atomic force microscopy (AFM). The dislocations that we observed may also create spirals on the surface in most cases. Fig. 6 is a schematic representation of a curved dislocation. A curved dislocation is used in this drawing just to present all the 
possible angles between the dislocation line and its Burgers vector. The Burgers vector is vertical; thus the dislocation is pure screw at end $\mathrm{S}$, pure edge at end $\mathrm{E}$, and mixed at any intermediate point such as M. Let us draw two Burgers circuits near S and M. By this we draw a closed circuit around the line as shown in perspective in Figs. 7 and 8. Assume now the presence of a surface perpendicular to the line of the dislocation represented by arrow $\mathbf{l}$ in the figures. From Figs. 7 and 8 , one understands that the surface would present a kink so that a spiral would be the trace of the dislocation on this surface. In the case of the screw dislocation, this spiral is circular, while in the case of a mixed dislocation, it is elongated in the direction of the inclination of the line. One may notice (Malkin et al., 1999; Yoshizaki et al., 2001) that the figures are elongated. These simple drawings explain why it is very dangerous to try to characterize dislocations only by surface studies.

\section{Conclusion}

HEW crystals have been studied by double-crystal synchrotron topography. The topographs show that the quality of the crystals is very high. They reveal the existence of long-range deformations, linear and two-dimensional defects: dislocations, growth band, intermediate growth surfaces and sector boundaries.

Double-crystal topography is a high-precision diffraction method which is very sensitive to the nature of the defects and which allows a quantitative analysis of their features. Using weak beam conditions, we have been able to characterize fully the growth dislocations roughly perpendicular to the $\{110\}$ faces as mostly edge dislocations with $\langle 001\rangle$ Burgers vectors. Their energy is lower than the energy of equivalent screw dislocations growing along the same direction because the

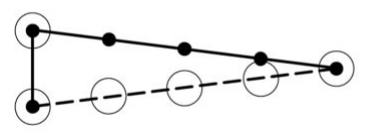

(a)

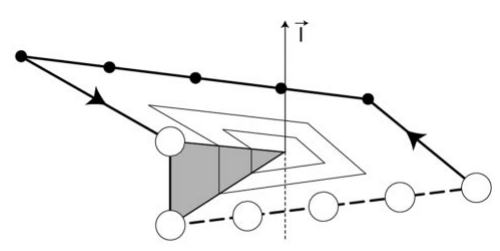

(b)

\section{Figure 7}

Drawing of a Burgers circuit around the core of the dislocation (line $\mathbf{1}$ ). On the left the circuit is shown as in Fig. 6 near point $S$; on the right is a perspective drawing (a)
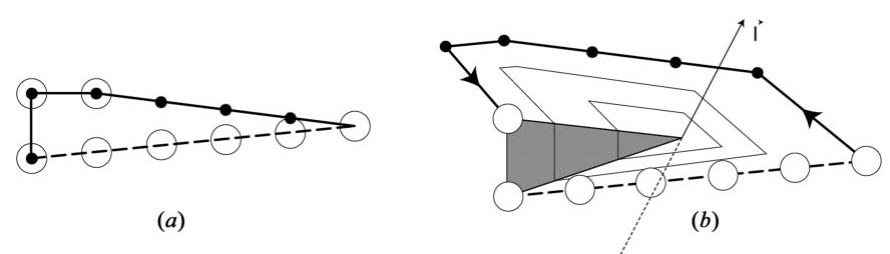

Figure 8

Same drawing of the Burgers circuit as in Fig. 7 but as shown near point $M$ in Fig. 6. length of their Burgers vector is much shorter. Although less favourable for the growth of the $\{110\}$ faces, this explains the absence of screw dislocations.

We have also explained why surface methods are unable to distinguish between screw and mixed dislocations, except in the very special case of a pure screw exactly perpendicular to the faces.

These various defects are well known in solution-grown crystals and these biological crystals do not reveal any special features. This means that all the expertise acquired in the study of the growth of mineral crystals in solution may be used to enhance the quality of biological crystals.

We are very indebted to Dr M. C. Robert who kindly helped us to examine the topographs and to analyse the various features of their contrast.

\section{References}

Authier, A. (1987). Adv. X-ray Anal. 10, 9-131.

Baruchel, J. (2000). http://www.esrf.fr/exp_facilities/ID19/homepage/ ID19GeneralPresentation.htm.

Baruchel, J., Härtwig J. \& Pernot-Rejmánková, P. (2002). J. Synchrotron Rad. 9, 107-114.

Bowen, D. K. \& Tanner, B. K. (1998). High Resolution X-ray Diffractometry and Topography. London: Taylor and Francis.

Carter, C. W. Jr \& Sweet, R. M. (1997). Methods in Enzymology, Macromolecular Crystallography, Vol. 276. New York: Academic Press.

Dobrianov, I., Caylor, C., Lemay, L. G., Finkelstein, K. D. \& Thorne, R. E. (1999). J. Cryst. Growth, 196, 511-523.

Durbin, S. D. \& Feher, G. (1986). J. Cryst. Growth, 76, 583-592.

Fourme, F., Ducruix, A., Riess-Kautt, M. \& Capelle, B. (1995). J. Synchrotron Rad. 2, 136-142.

Gits-Leon, S., Lefaucheux, F. \& Robert, M. C. (1978). J. Cryst. Growth, 44, 345-355.

Izumi, K., Sawamura, S. \& Ataka, M. (1996). J. Cryst. Growth, 168 106-111.

Izumi, K., Taguchi, K., Kobayashi, K., Tachibana, M., Kojima, K. \& Ataka, M. (1999). J. Cryst. Growth, 206, 155-158.

Lefaucheux, F., Robert, M. C. \& Authier, A. (1973). J. Cryst. Growth, 19, 329-337.

Malkin, A. J., Kuznestov, Y. G. \& McPherson, A. (1999). J. Cryst. Growth, 196, 471-488.

Otálora, F., Capelle, B., Ducruix, A. \& Garcìa-Ruiz, J. M. (1999). Acta Cryst. D55, 644-649.

Otálora, F., Garcìa-Ruiz, J. M., Gavira, J. A. \& Capelle, B. (1999). J. Cryst. Growth, 196, 546-558.

Otálora, F., Gavira, J. A., Capelle, B. \& Garcìa-Cruiz, J. M. (1999). Acta Cryst. D55, 650-655.

Otwinowski, Z. \& Minor, W. (1997). Processing of X-ray Diffraction Data Collected in Oscillation Mode, Methods in Enzymology, Macromolecular Crystallography, Vol. 276, part A, pp. 307-326. New York: Acadenic Press.

Stojanoff, V. \& Siddons, D. P. (1996). Acta Cryst. A52, 498-499.

Stojanoff, V., Siddons, D. P., Monaco, L. A., Vekilov, P. \& Rosenberg, P. (1997). Acta Cryst. D53, 588-595.

Strunk, H. P. (1996). J. Cryst. Growth, 160, 184-185.

Yoshizaki, I., Sato, T., Igarashi, N., Natsuisaka, M., Tanaka, N., Komatsu, H. \& Yoda, S. (2001). Acta Cryst. D57, 1621-1629. 